\section{JTI}

JOURNAL OF

TRAUMA AND INJURY

\title{
Large Focal Extrapleural Hematoma of Chest Wall: A Case Report
}

\author{
Hohyoung Lee, M.D., Sung Ho Han, M.D., Min Koo Lee, M.D., Ph.D., \\ Oh Sang Kwon, M.D., Kyoung Hwan Kim, M.D., Jung Suk Kim, M.D., \\ Soon-Ho Chon, M.D., Ph.D., Sung Ho Shinn, M.D., Ph.D. \\ Departments of Trauma Surgery and Thoracic and Cardiovascular Surgery, Cheju Halla \\ Hospital, Jeju, Korea
}

\begin{abstract}
Although hemothorax and pneumothorax are common complications seen in rib fractures, focal extrapleural hematoma is quite rare. We report a 63-year-old female patient that developed large focal extrapleural hematoma after falling off a second floor veranda. The patient had sustained 3, 4, 5th costal cartilage rib fractures and a sternum fracture. She had developed suspected empyema with loculations with small amount of hemothorax. She underwent a planned early decortication/adhesiolysis by video assisted thoracoscopic surgery at the 12th post-trauma day due to failed drainage. Unexpectedly, she had no adhesions or any significant retained hematoma mimicking a mass, but was found with the focal extrapleural chest wall hematoma. She was discharged on postoperative 46th day for other reasons and is doing fine today.
\end{abstract}

Keywords: Hemothorax; Blunt injury; Video assisted thoracoscopic surgery

\section{INTRODUCTION}

Hemothorax is not a rare phenomenon after rib fractures in blunt chest wall injury, however, a large focal extrapleural hematoma is not seen as frequently. Usually a chest tube or low caliber drain may resolve posttraumatic hemothoraces, however, large extrapleural hematomas would have to be treated surgically [1]. Lately, treatment can be done in a minimaly invasive manner [2]. We report a case of large focal extrapleural hematoma after fall down injury in a patient who had sustained minor rib cartilage fractures and sternum fractures after fall down. The typical "stripe signs" were not easily seen on chest computer tomography [3]. She underwent successful minimally invasive thoracoscopic surgery after failed chest drainage. The patient was discharged on the postoperative 46th day with resolution and is happy with the results. 


\section{CASE REPORT}

A 63 year-old female patient had visited our emergency center at Halla Hospital after fall down injury from an apartment veranda on the second floor with symptoms of chest wall pain, back pain and lower extremity pain. She was found with multiple rib cartilage fractures from her right 3 rd to 5 th ribs, sternum fracture and moderate amount of right hemothorax, L2 unstable burst fracture, L1 compression fracture, left transverse process fractures of L1, L2, and L4, superior rami fracture of her left hip, and right calcaneus fracture. Her initial computer tomogram presented the focal hematoma (Fig. 1). Her initial hemoglobin levels

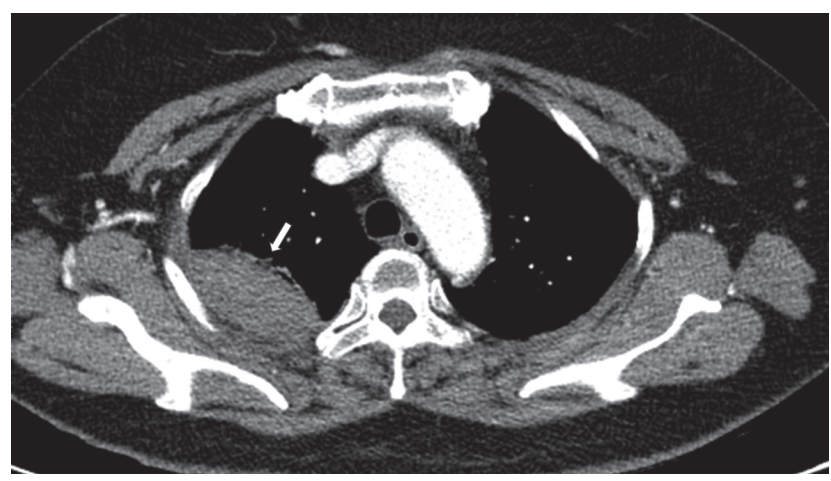

Fig. 1. Initial computer tomogram of the patient showing the focal hematoma (arrow).

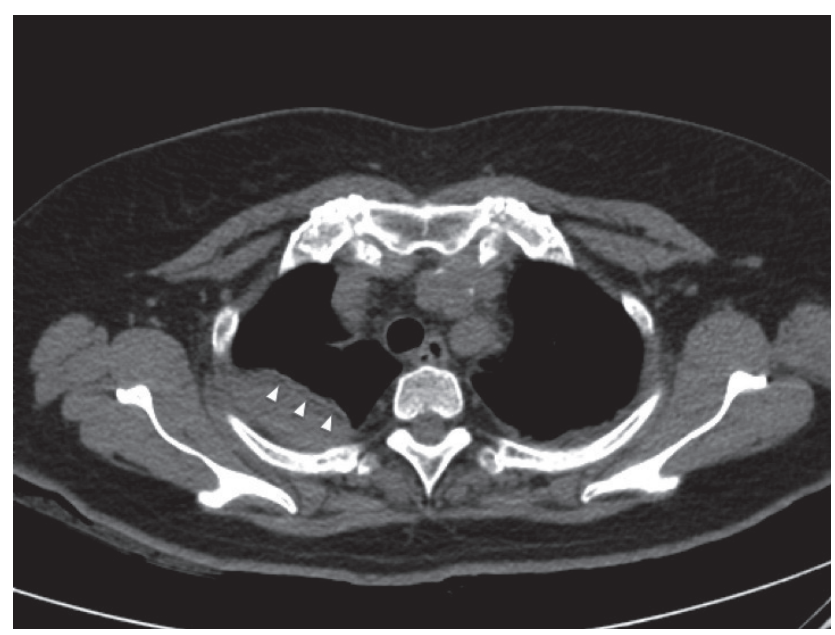

Fig. 2. Computer tomogram of the patient's chest showing large amount of right extrapleural hematoma presenting the "stripe" sign (arrowheads) taken 4 days later. were $12.8 \mathrm{~g} / \mathrm{dL}$, but dropped to $9.5 \mathrm{~g} / \mathrm{dL}$ the day after. Two pints of blood were given. Pig-tail drainage was done on the 4th hospital day without resolution and a follow up chest computer tomogram was taken. The images presented remaining local hematoma (Fig. 2). Thus, a video assisted thoracoscopic procedure was scheduled on the 11th hospital day with supposed diagnosis of loculated effusion or impending empyema. The procedure was done to allow re-expansion of her lung.

The procedure was done with two ports, with $5 \mathrm{~mm}$ camera port in her 9th intercostal space in her mid-axillary line and a $5 \mathrm{~mm}$ port anteriorly. There were no adhesions and a large hematoma sac was seen at about $10 \times 8 \mathrm{~cm}$ in dimensions with dark blood discoloration (Fig. 3). The sac was opened with Harmonic scalpel at the base and at its entire longitudinal length. The procedure

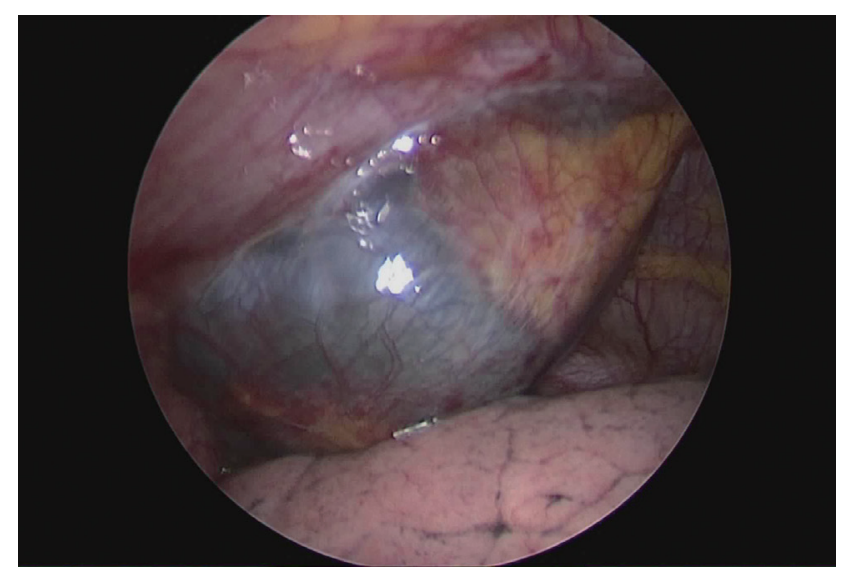

Fig. 3. Intraoperative view of the large extrapleural hematoma as seen in a video captured image.
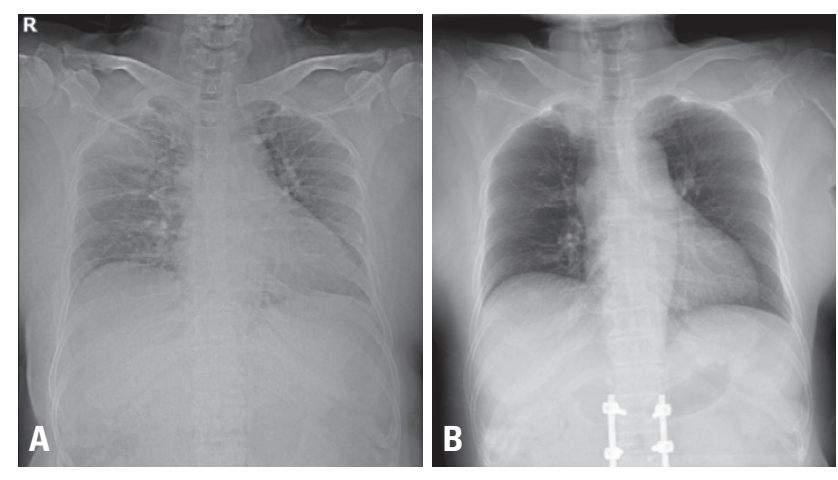

Fig. 4. Initial simple chest $X$-ray (A) and postoperative simple chest X-ray (B). 
was completed after placing a 24 Fr. chest tube.

She underwent L-spine fusion for her unstable L2 fracture and open reduction and internal fixation of her calcaneus three days after the thoracoscopic procedure. The tube was removed on the day after the orthopedic procedure. She was discharged on the 46th hospital day after rehabilitation and is doing well today 9 months after her injury (Fig. 4).

\section{DISCUSSION}

Focal large extrapleural hematoma after blunt trauma with minor rib fractures is a rare event, but can prove to be hemodynamically challenging [3]. Diagnosis can only be confirmed by a chest computer tomogram. Classical signs present a "stripe" seen on cross-sectional computer tomographic scans. This sign is also called the "displaced extrapleural fat sign" caused by intercostal vessel bleeding [3]. Our particular case presented a very faint stripe sign.

The incidence of a extrapleural hematoma after blunt trauma has been reported to be as high as 7.1\% [1]. Manifestations may include chest pain in high-impact trauma that can lead to delayed hemorrhagic shock [4]. Absence of displaced rib fractures on simple X-rays does not exclude such hematomas and if hypovolemic or hemorrhagic shock ensues, further evaluation is necessary. In our case, a computer tomogram of her chest helped delineate origin of bleeding (the hematoma site).

Angiographic embolization would provide an attractive treatment option and could preclude a more invasive interventional procedure. However, this may not provide for adequate drainage and waiting for absorption could take time. There is a successful case of one such patient with extrapleural hematoma treated by embolization and pig-tail drainage [5]. Thoracotomy was done in over half the patients with significant sized extrapleural hematomas [1]. Thoracoscopic surgery is an attractive option when it comes to surgery. In other words, it would be better to include a minimally invasive procedure in the condition that an invasive procedure should be performed.

An ongoing actively bleeding large extrapleural hematoma would necessitate bleeding control. This can be done through angiographic embolization and drainage or a surgical procedure. Early diagnosis and awareness of such an entity could be life-saving. Thoracoscopic drainage and bleeding control could provide an attractive option to treatment of large extrapleural hematomas after blunt trauma.

\section{REFERENCES}

1. Rashid MA, Wikström T, Ortenwall P. Nomenclature, classification, and signficance of traumatic extrapleural hematoma. J Trauma 2000:49:286-90.

2. Narsule CK, Sarkar B. Thoracoscopic evacuation of giant traumatic extrapleural hematomas. J Trauma Acute Care Surg 2017;82:963-5.

3. Ezaki J, Ro A, Kibayashi K. The histopathological structures of the extrapleural hematoma wall: a case report. Forensic Sci Int 2016;266:e68-70.

4. Sato N, Sekiguchi H, Hirose Y, Yoshida S. Delayed chest wall hematoma caused by progressive displacement of rib fractures after blunt trauma. Trauma Case Rep 2016;4:1-4.

5. Gorospe L, Fernández-Méndez MÁ, Ayala-Carbonero A, Cabañero-Sánchez A, Muñoz-Molina GM. Nonoperative management of a large extrapleural hematoma after blunt chest trauma. J Emerg Med 2016;51:159-63. 